\title{
Vascular damage and EEG markers in subjects with mild cognitive impairment
}

\author{
D.V. Moretti ${ }^{a, *}$, C. Miniussi ${ }^{\mathrm{a}, \mathrm{b}}$, G. Frisoni ${ }^{\mathrm{a}}$, O. Zanetti ${ }^{\mathrm{a}}$, G. Binetti ${ }^{\mathrm{a}}$, \\ C. Geroldi ${ }^{a}$, S. Galluzzi a , P.M. Rossini ${ }^{\mathrm{a}, \mathrm{c}, \mathrm{d}}$ \\ a IRCCS 'S. Giovanni di Dio - Fatebenefratelli', 4, Pilastroni Road, 25125 Brescia, Italy \\ ${ }^{\mathrm{b}}$ Department of Biomedical Sciences and Biotechnologies, University of Brescia, Italy \\ ' AFaR Department of Neuroscience, 'S. Giovanni Calibita-Fatebenefratelli', Rome, Italy \\ d Clinical Neurology, University 'Campus Biomedico', Rome, Italy
}

Accepted 8 May 2007

\begin{abstract}
Objective: We evaluated the changes induced by cerebrovascular $(\mathrm{CV})$ damage on brain rhythmicity recorded by electroencephalography (EEG) in a cohort of subjects with mild cognitive impairment (MCI).

Methods: We enrolled 99 MCI subjects (Mini-Mental State Examination [MMSE] mean score 26.6). All subjects underwent EEG recording and magnetic resonance imaging (MRI). EEGs were recorded at rest. Individual EEG frequencies were indexed by the $\theta / \alpha$ transition frequency (TF) and by the individual $\alpha$ frequency (IAF) with power peak in the extended $\alpha$ range (5-14 Hz). Relative power was separately computed for $\delta, \theta, \alpha 1, \alpha 2$, and $\alpha 3$ frequency bands on the basis of the TF and IAF values. Subsequently, we divided the cohort in four sub-groups based on subcortical CV damage as scored by the age-related white matter changes scale (ARWMC).

Results: CV damage was associated with 'slowing' of TF proportional to its severity. In the spectral bandpower the severity of vascular damage was associated with increased $\delta$ power and decreased $\alpha 2$ power. No association of vascular damage was observed with IAF and $\alpha 3$ power. Moreover, the $\theta / \alpha 1$ ratio could be a reliable index for the estimation of the individual extent of CV damage.

Conclusions: EEG analysis may show physiological markers sensitive to CV damage. The appropriate use of this EEG index may help the differential diagnosis of different forms of cognitive decline, namely primary degenerative and secondary to CV damage.

Significance: The EEG neurophysiological approach, together with anatomical features from imaging, could be helpful in the understanding of the functional substrate of dementing disorders.

(C) 2007 International Federation of Clinical Neurophysiology. Published by Elsevier Ireland Ltd. All rights reserved.
\end{abstract}

Keywords: Mild cognitive impairment; Vascular cognitive impairment; Electroencephalography; $\alpha$ Rhythm; MRI; ARWMC scale

\section{Introduction}

The role of cerebrovascular $(\mathrm{CV})$ disease and ischemic brain damage in cognitive decline remains controversial. Although not all patients with mild cognitive impairment due to $\mathrm{CV}$ damage develop a clinically defined dementia, all such patients are at risk and could develop dementia in the 5 years following the detection of cognitive decline. According to the studies (Wentzel et al., 2001; Gorelick,

\footnotetext{
* Corresponding author. Tel.: +39 0303501597; fax: +390303533513.

E-mail address: davide.moretti@afar.it (D.V. Moretti).
}

2003), the percentage of these patients varies from $25 \%$ to $50 \%$. Cognitive impairment due to subcortical CV damages is thought to be caused by focal or multifocal lesions involving strategic brain areas. These lesions in basal ganglia, thalamus or connecting white matter induce interruption of thalamocortical and striatocortical pathways. As a consequence, deafferentation of frontal and limbic cortical structures is produced. The pattern of cognitive impairment is consistent with models of impaired cortical and subcortical neuronal pathways (Kramer et al., 2002). Complex interactions in producing cognitive decline have been shown (Fein et al., 2000) between subcortical lesions 
and changes in cortex and hippocampus, while other interactions suggested significant correlations between cortical lesions and frontal atrophy (Burton et al., 2003; Hentschel et al., 2003). Preclinical (Kimura et al., 2000) and clinical evidence (Swartz and Black, 2002) indicate that a cholinergic deficit similar to that seen in Alzheimer's dementia (AD) may be associated with vascular dementia (VaD); which suggests that these patients may benefit from treatment with cholinesterase inhibitors, as has been confirmed in recent studies (Erkinjuntti et al., 2000).

Morphological substrates of cognitive impairment resulting from $\mathrm{CV}$ lesions remain confusing, in that they constitute a multifactorial disorder related to a wide variety of lesions and causes. Even when CV pathology appears to be the main underlying process, the effects of the damaged brain parenchyma are variable and, therefore, the clinical, radiological and pathological appearances may be heterogeneous. Mild cognitive impairment (MCI) is a clinical status intermediate between elderly normal cognition and dementia, and characterized by memory complaints and cognitive impairment, but not by dementia, on neuropsychological testing (Flicker et al., 1991; Petersen et al., 1995, 2001). MCI is a clinically heterogeneous syndrome comprising a number of conditions (Petersen et al., 2001; Portet et al., 2006). Among these, amnestic MCI (aMCI), characterized by memory deficit and preserved general cognition, is the most clearly defined. Anyway, the natural history of a group of subjects at very high-risk for developing dementia due to subcortical vascular damage [subcortical vascular MCI (svMCI)] has recently been described (Frisoni et al., 2002; Galluzzi et al., 2005). In such study, MCI patients with CV etiology developed a distinctive clinical phenotype characterized by poor performance on frontal tests, and neurological features of parkinsonism without tremor (impairment of balance and gait).

A neurophysiological approach could be helpful in differentiating structural from functional $\mathrm{CV}$ damage. The quantitative analysis of electroencephalographic (EEG) rhythms in resting subjects is a low-cost but still powerful approach to the study of elderly subjects in normal aging, MCI and dementia (Gueguen et al., 1991; Maurer and Dierks, 1992; Leuchter et al., 1993; Schreiter-Gasser et al., 1993; Zappoli et al., 1995; Jelic et al., 1996, 2000; Huang et al., 2000; Bennys et al., 2001; Koenig et al., 2005; Babiloni et al., 2006a,b; Prichep et al., 2006; Rossini et al., 2006). The anchor point for a quantitative analysis of the EEG is the $\alpha$ rhythm, which dominates the EEG power spectrum in resting, awake and healthy subjects (Elul, 1972; Lopes da Silva et al., 1976, 1980; Steriade and Llinas, 1988; Singer, 1993; Destexhe and Sejnowski, 1996; Klimesch, 1997, 1999; Pfurtscheller and Lopes da Silva, 1999; Nunez et al., 2001; Suffczynski et al., 2001; Klimesch et al., 2007). Traditionally, the $\alpha$ power is evaluated in one or two fixed frequency bands ranging from 8 to $13 \mathrm{~Hz}$. Moreover, the analysis of individual EEG frequency bands (Kopruner et al., 1984; Niedermayer, 1993; Klimesch, 1999) could reveal different sub-bands in the range of the $\alpha$ frequency possibly supporting different cognitive functions (Moretti et al., 2004).

Our study aims at extending these results to subjects with MCI based on CV damage. Recently, it has been shown that individual $\alpha$-frequency markers and spectral power may capture different component (neural) processes, with these two indices discriminating between mild AD and VaD patients (Moretti et al., 2004). The study reported a reduction in EEG $\alpha$ power in AD patients which was not associated with a proportional change in $\alpha$ frequency, whereas patients with $\mathrm{VaD}$, in whom there were diffuse vascular lesions of the white matter, showed a slowing of the $\alpha$ frequency with minor change in the $\alpha$ power.

To the best of our knowledge, the relationship between vascular damage and EEG rhythms has not yet been explored in MCI subjects. In this study, we test the hypothesis that such a relationship does exist, not only in $\mathrm{VaD}$ as compared to AD patients, but also in a population of MCI subjects with an early cognitive impairment which is, however, homogeneous with respect to their cognitive decline. Furthermore, in this study we attempt to find specific electroencephalographic markers for vascular damage that could help in differential diagnosis between "pure" degenerative and vascular variants of dementing disorder.

\section{Materials and methods}

\subsection{Subjects}

For the present study, 99 subjects with MCI were recruited. All experimental protocols had been approved by the local Ethics Committee. Informed consent was obtained from all participants or their caregivers, according to the Code of Ethics of the World Medical Association (Declaration of Helsinki).

\subsection{Diagnostic criteria}

In this study we enrolled subjects afferent to the Scientific Institute of Research and Cure (IRCCS) 'Fatebenefratelli' in Brescia, Italy. Patients were taken from a prospective project on the clinical progression of MCI. The project was aimed to study the natural history of non-demented persons with apparently primary cognitive deficits not caused by psychic (anxiety, depression, etc.) or physical (uncontrolled heart disease, uncontrolled diabetes, etc.) conditions. Patients were rated with a series of standardized diagnostic tests, including the Mini-Mental State Examination (MMSE; Folstein et al., 1975), the Clinical Dementia Rating Scale (CDRS; Hughes et al., 1982), the Hachinski Ischemic Scale (HIS; Rosen et al., 1980), and the Instrumental and Basic Activities of Daily Living (IADL, BADL, Lawton and Brodie, 1969). In addition, patients underwent diagnostic neuroimaging procedures (magnetic resonance imaging, MRI) and laboratory blood analyses to rule out other causes of cognitive impairment. 
Our inclusion and exclusion criteria for MCI were based on previous seminal studies (Albert et al., 1991; Devanand et al., 1997; Flicker et al., 1991; Petersen et al., 1995, 1997, 2001; Portet et al., 2006; Geroldi et al., 2006). Study inclusion criteria were all of the following: (i) complaint by the patient, or report by a relative or the general practitioner, of memory or other cognitive disturbances; (ii) Mini-Mental State Examination (MMSE; Folstein et al., 1975) score of 24 to 27/30, or MMSE of 28 and higher plus low performance (score of $2 / 6$ or higher) on the clock drawing test (Shulman, 2000); (iii) sparing of instrumental and basic activities of daily living, or functional impairment stably due to causes other than cognitive impairment, such as physical impairments, sensory loss, gait or balance disturbances, etc. Exclusion criteria were any one of the following: (i) age of 90 years and older; (ii) history of depression or psychosis of juvenile onset; (iii) history or neurological signs of major stroke; (iv) other psychiatric diseases, epilepsy, drug addiction, alcohol dependence; (v) use of psychoactive drugs including acetylcholinesterase inhibitors or other drugs enhancing brain cognitive functions; and (vi) current or previous uncontrolled or complicated systemic diseases (including diabetes mellitus) or traumatic brain injuries.

All patients underwent: (i) semi-structured interview with the patient and - whenever possible - with another informant (usually the patient's spouse or a child) by a geriatrician or neurologist; (ii) physical and neurological examinations; (iii) performance-based tests of physical function, gait and balance; (iv) neuropsychological assessment evaluating verbal and non-verbal memory, attention and executive functions (Trail Making Test B-A; Clock Drawing Test; Amodio et al., 2002; Shulman, 2000), abstract thinking (Raven matrices; Basso et al., 1987), frontal functions (Inverted Motor Learning; Spinnler and Tognoni, 1987); language (Phonological and Semantic fluency; Token test; Carlesimo et al., 1996; Novelli et al., 1986), and apraxia and visuo-constructional abilities (Rey figure copy; Caffarra et al., 2002); (v) assessment of depressive symptoms with the Center for Epidemiologic Studies Depression Scale (CES-D; Radloff, 1977). As the aim of our study was to evaluate the impact of the vascular damage on EEG rhythms, we did not consider the clinical subtype of MCI, i.e., amnesic, non-amnesic or multiple domains.

\subsection{EEG recordings}

All recordings were obtained in the morning with subjects resting comfortably. Vigilance was continuously monitored in order to avoid drowsiness.

The EEG activity was recorded continuously from 19 sites by using electrodes set in an elastic cap (Electro-Cap International, Inc.) and positioned according to the 10-20 International system (Fp1, Fp2, F7, F3, Fz, F4, F8, T3, C3, Cz, C4, T4, T5, P3, Pz, P4, T6, O1, O2). The ground electrode was placed in front of Fz. The left and right mastoids served as reference for all electrodes. The recordings were used offline to rereference the scalp recordings to the common average. Data were recorded with a band-pass filter of $0.3-70 \mathrm{~Hz}$, and digitized at a sampling rate of $250 \mathrm{~Hz}$ (BrainAmp, BrainProducts, Germany). Electrodes-skin impedance was set below $5 \mathrm{k} \Omega$. Horizontal and vertical eye movements were detected by recording the electrooculogram (EOG). The recording lasted $5 \mathrm{~min}$, with subjects with closed eyes. Longer recordings would have reduced the variability of the data, but they would also have increased the possibility of slowing of EEG oscillations due to reduced vigilance and arousal. EEG data were then analyzed and fragmented off-line in consecutive epochs of $2 \mathrm{~s}$, with a frequency resolution of $0.5 \mathrm{~Hz}$. The average number of epochs analyzed was 140 (range, about 130-150).

The EEG epochs with ocular, muscular and other types of artifacts were discarded.

\subsection{Analysis of individual frequency bands}

A digital FFT-based power spectrum analysis (Welch technique, Hanning windowing function, no phase shift) computed - ranging from 2 to $40 \mathrm{~Hz}$ - the power density of EEG rhythms with a $0.5 \mathrm{~Hz}$ frequency resolution. Two anchor frequencies were selected according to literature guidelines (Klimesch, 1999), that is, the $\theta / \alpha$ transition frequency (TF) and the individual $\alpha$ frequency (IAF) peak. As previously said, the TF marks the transition frequency between the $\theta$ and $\alpha$ bands, and represents an estimate of the frequency at which the $\theta$ and $\alpha$ spectra intersect. We computed the TF as the minimum power in the $\alpha$ frequency range, since our EEG recordings were performed at rest. The IAF represents instead the frequency with the maximum power peak within the extended $\alpha$ range $(5-14 \mathrm{~Hz})$. TF and IAF could be clearly identified in 99 MCI subjects whose EEG data were then statistically analyzed. Based on TF and IAF, we estimated the frequency band range for each subject, as follows: $\delta$ from TF-4 to TF-2, $\theta$ from TF-2 to TF, low $\alpha$ band $(\alpha 1$ and $\alpha 2$ ) from TF to IAF, and high $\alpha$ band (or $\alpha 3$ ) from IAF to IAF +2 . The $\alpha 1$ and $\alpha 2$ bands were computed for each subject as follows: $\alpha 1$ from TF to the middle point of the TF-IAF range, and $\alpha 2$ from such middle point to the IAF peak (Moretti et al., 2004). We found that the bandwidth in $\alpha 1$ and $\alpha 2$ bands differed among the groups. In the group without vascular damage, as well as in the group with severe vascular damage, the bandwidth was slightly narrower $(1.48$ and $1.53 \mathrm{~Hz}$, respectively) than in the groups with mild and moderate vascular damage (1.7 and $1.87 \mathrm{~Hz}$, respectively). We performed a statistical analysis to test whether this difference was significant among groups in these frequency bands. However, the analysis did not show a significant main group effect $(p=0.06)$. Finally, in the frequency bands determined in this way, we computed the relative power spectra for each subject. The relative power density for each frequency band was computed as the ratio between 
the absolute power and the mean power spectra from 2 to $40 \mathrm{~Hz}$. The relative band power at each band was defined as the mean of the relative band power for each frequency bin within that band.

\subsection{Magnetic resonance imaging (MRI) and $C V$ damage evaluation}

Magnetic resonance (MR) images were acquired by using a 1.0 Tesla Philips Gyroscan. Axial T2-weighted, proton density-weighted imaging (ProWI) and fluid attenuated inversion recovery (FLAIR) images were acquired with the following acquisition parameters: $\mathrm{TR}=2000 \mathrm{~ms}$, $\mathrm{TE}=8.8 / 110 \mathrm{~ms}$, flip angle $=90^{\circ}$, field of view $=230 \mathrm{~mm}$, acquisition matrix $256 \times 256$, slice thickness $5 \mathrm{~mm}$ for T2/ ProWI sequences and TR $=5000 \mathrm{~ms}, \mathrm{TE}=100 \mathrm{~ms}$, flip angle $=90^{\circ}$, field of view $=230 \mathrm{~mm}$, acquisition matrix 256x256, slice thickness $5 \mathrm{~mm}$ for FLAIR images.

Subcortical cerebrovascular disease (sCVD) was assessed by using the rating scale for age-related white matter changes (ARWMC) on T2-weighted and FLAIR MR images. White matter changes (WMC) were rated by a single observer (R.R.) in the right and left hemispheres, separately, in frontal, parieto-occipital, temporal, infratentorial areas and basal ganglia on a 4-point scale. The observer of white matter changes was blind to the clinical information on the subjects. Subscores of $0,1,2$, and 3 were assigned in frontal, parieto-occipital, temporal, infratentorial areas for: no WMC, focal lesions, beginning confluence of lesions, and diffuse involvement of the entire region, respectively. Subscores of $0,1,2$, and 3 were assigned in basal ganglia for: no WMC, 1 focal lesion, more than 1 focal lesion and confluent lesions, respectively. The total score was the sum of the subscores for each area in the left and right hemispheres (range, 0-30). With respect to the ARWMC scale, the interrater reliability, as calculated with weighted $\mathrm{k}$ value, was 0.67 , which was indicative of moderate agreement (Wahlund et al., 2001). We assessed test-retest reliability on a random sample of 20 subjects. The intraclass correlation coefficient was 0.98 , values above 0.80 being considered indicative of good agreement.

Based on increasing subcortical CV damage, the $99 \mathrm{MCI}$ subjects were subsequently divided into four sub-groups along the range between the minimum and maximum ARWMC scores (respectively, 0 and 15). The first group was composed by subjects with score $=0$, so that the highest sensitivity to the $\mathrm{CV}$ damage could be obtained. The other groups were composed according to equal-range ARWMC scores. As a consequence, we obtained the following groups: group 1 (G1), no vascular damage, $\mathrm{CV}$ score 0 ; group 2 (G2), mild vascular damage, $\mathrm{CV}$ score 1-5; group 3 (G3), moderate vascular damage, CV score 6-10; group four (G4), severe vascular damage, $\mathrm{CV}$ score 11-15.

Table 1 shows the mean values of the demographic and clinical characteristics of the four sub-groups.
Table 1

Mean values \pm standard error of demographic characteristics, neuropsychological and ARWMC scores of the MCI sub-groups

\begin{tabular}{lcccl}
\hline & Group 1 & Group 2 & Group 3 & Group 4 \\
\hline Subjects (F/M) & $27(18 / 9)$ & $41(31 / 10)$ & $19(10 / 9)$ & $12(9 / 3)$ \\
Age & $70.1( \pm 1.7)$ & $69.9( \pm 1.1)$ & $69.7( \pm 1.9)$ & $70.5( \pm 2.4)$ \\
Education & $7.1( \pm 0.7)$ & $7( \pm 0.6)$ & $7( \pm 0.9)$ & $10( \pm 1.6)$ \\
MMSE & $26.7( \pm 0.4)$ & $26.5( \pm 0.4)$ & $27( \pm 0.4)$ & $26.1( \pm 0.7)$ \\
ARWMC scale & 0 & $1-5$ & $6-10$ & $11-15$ \\
\hline
\end{tabular}

$\mathrm{F} / \mathrm{M}$, female/male. Age and education are expressed in years.

Group 1, no vascular damage; group 2, mild vascular damage; group 3, moderate vascular damage; group 4, severe vascular damage.

\subsection{Statistical analysis}

Preliminarily, any significant differences between groups in demographic variables, i.e., age, education and gender, as well as MMSE score, were taken into account. Only education showed a significant difference between groups $(p<0.03)$. In order to avoid a confounding effect, subsequent statistical analyses of variance (ANOVAs) were carried out using age, education, gender and MMSE score as covariates. Duncan's test was used for post-hoc comparisons. For all statistical tests the significance was set at $p<0.05$.

First, addressing the issues of the "shifting" and, consequently, the "slowing" of EEG, we performed a first session of ANOVAs to compare frequency markers. In the first analysis, the TF was the dependent variable; in the second analysis, the IAF was the dependent variable, while, in the third analysis, TF and IAF were analyzed together as dependent variable. The Group factor was the independent variable in each analysis.

A second session of ANOVA was performed on EEG relative power data. In this analysis, the Group factor was the independent variable and the frequency band power $(\delta, \theta, \alpha 1, \alpha 2, \alpha 3)$ was the dependent variable.

As a successive step, and in order to evaluate the presence of EEG indices that specifically correlated with the vascular damage, we performed statistical analyses to assess the specificity of the following ratios: $\theta / \alpha 1$ (by using also the TF as a covariate); $\alpha 2 / \alpha 3$ (by using also the IAF as a covariate), and $\alpha 1 / \alpha 2$, with both TF and IAF as covariates. Moreover, we performed correlations (Pearson's moment correlation) between $\mathrm{CV}$ damage score and frequency markers (TF and IAF), spectral power, and MMSE. Finally, we performed a control statistical analysis with four frequency bands, considering $\alpha 1$ and $\alpha 2$ as single bands (low- $\alpha$ band). This analysis aimed to verify whether the low $\alpha$ band, when considered as a whole, had the same behaviour.

\section{Results}

Table 2 reports means and standard errors for IAF and TF in the 4 sub-groups of MCI subjects. 
Table 2

Mean values \pm standard error of individual $\alpha$ frequency (IAF) and transition frequency (TF) in the MCI sub-groups

\begin{tabular}{lllll}
\hline & Group 1 & Group 2 & Group 3 & Group 4 \\
\hline IAF & $9.1 \pm 0.2$ & $9.3 \pm 0.1$ & $9.13 \pm 0.2$ & $9.6 \pm 0.4$ \\
TF & $6.5 \pm 0.2$ & $6.1 \pm 0.1$ & $5.9 \pm 0.2$ & $5.5 \pm 0.3$ \\
\hline
\end{tabular}

Group 1, no vascular damage; group 2, mild vascular damage; group 3, moderate vascular damage; group 4 , severe vascular damage.

In the analysis of frequency markers, when TF and IAF were analyzed individually as dependent variables, we found no significant main effect $(F(3.91)=2.07 ; p=0.1$ and $F(3.91)=.38 ; p=0.7$, respectively).

On the contrary, the analysis revealed significant interactions between Group and both TF and IAF as dependent variables $[F(3.95)=3.70 ; p<0.010]$. Specifically, Duncan's post-hoc test showed a significant TF slowing between G1 and all the other groups, as well as between G2 and G4 (Fig. 1). Individual frequency bands were selected on the basis of TF and IAF, and the corresponding relative power density was computed by collapsing the spectra. Fig. 2 displays the results of the ANOVA of these data showing a significant interaction between Group and Band factors $[F(12.380)=2.60 ; \quad p<0.002] . \quad$ Interestingly, Duncan's post-hoc test showed a significant decrease in the $\delta$ power in $\mathrm{G} 1$, as compared to $\mathrm{G} 4(p<0.050)$, and a significant increase in the $\alpha 2$ power in G1, as compared to G3 and G4 $(p<0.000)$. On the contrary, no differences were found in $\theta, \alpha 1$ and $\alpha 3$ power bands. Moreover, a closer look at the data relating to the $\alpha 1$ frequency showed a decrease - proportional to the degree of $\mathrm{CV}$ damage - which, although not significant, was very similar to that in the $\alpha 2$ band. Reversely, in the $\alpha 3$ power band, this trend was not present, which suggests that the vascular damage had no impact on this frequency band.
The correlation analysis between $\mathrm{CV}$ damage score and frequency indices (TF and IAF) showed a significant negative correlation of CV score and TF $(r=-0.243 ; p<0.01)$, whereas the correlation was not significant with IAF $(r=-0.006 ; p=0.9516)$.

The correlation analysis between $\mathrm{CV}$ damage score and spectral band power showed a significant positive correlation with the $\delta$ power $(r=0.221 ; p<0.03)$, and a significant negative correlation with the power of $\alpha 1$ $(r=-0.312 ; \quad p<0.002)$ and $\alpha 2$ frequency bands $(r=-0.363 ; p<0.0003)$. The correlations between $\mathrm{CV}$ damage score with the $\theta$ power $(r=0.183 ; p=0.07)$ and the $\alpha 3$ power $(r=-0.002 ; p=0.93)$ were not significant, as well as the correlation between $\mathrm{CV}$ damage score and $\operatorname{MMSE}(r=-0.07 ; p=0.4)$.

Table 3 displays the values of $\theta / \alpha 1$ and $\alpha 2 / \alpha 3$ power ratios. The statistical analysis of the $\theta / \alpha 1$ ratio showed a main effect of the Group factor $[F(3.91)=15.51$; $p<0.000]$. Duncan's post-hoc testing showed a significant increase in the $\theta / \alpha 1$ ratio between G1 and G2 with respect to $\mathrm{G} 3$ and $\mathrm{G} 4(p<0.000)$. Moreover, the increase in this ratio was significant also between G3 and G4 $(p<0.04)$. The statistical analysis of the $\alpha 2 / \alpha 3$ power ratio showed a main effect of the Group factor $[F(3.91)=4.60$; $p<0.005]$. Duncan's post-hoc testing showed a significant decrease in the ratio between G1 and G3 $(p<0.02)$, G1 and G4 $(p<0.010)$, and G2 and G4 $(p<0.05)$. The statistical analysis of the $\alpha 1 / \alpha 2$ ratio did not show the main effect of the Group factor $(p<0.2)$.

Fig. 3 displays the control analysis, performed to evaluate the low $\alpha$ as a whole. ANOVA showed a significant interaction between Group and Band factors $(F(9.285)=4.12 ; p<0.000)$. Duncan's post-hoc testing showed a significant decrease in the $\alpha$ power between G1 and both G3 and G4 $(p<0.0000)$, which confirms the

\footnotetext{
Plot of Means

2-way interaction

$F(3,95)=3,70 ; p<, 0143$
}

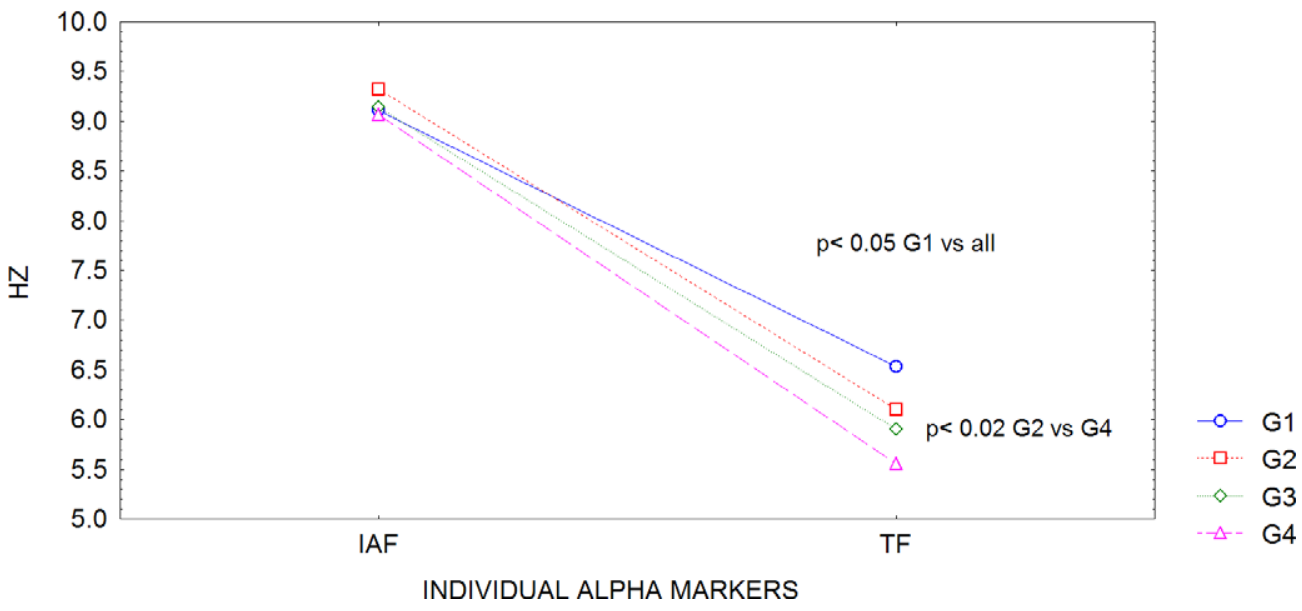

Fig. 1. Statistical ANOVA interaction among groups, factor (G1, G2, G3, G4) and individual alpha markers (TF and IAF). In the diagram the difference in TF among groups is also indicated, based on Duncan's post-hoc testing. (G1, group 1) no vascular damage; (G2, group 2) mild vascular damage; (G3, group 3) moderate vascular damage; (G4, group 4) severe vascular damage. 


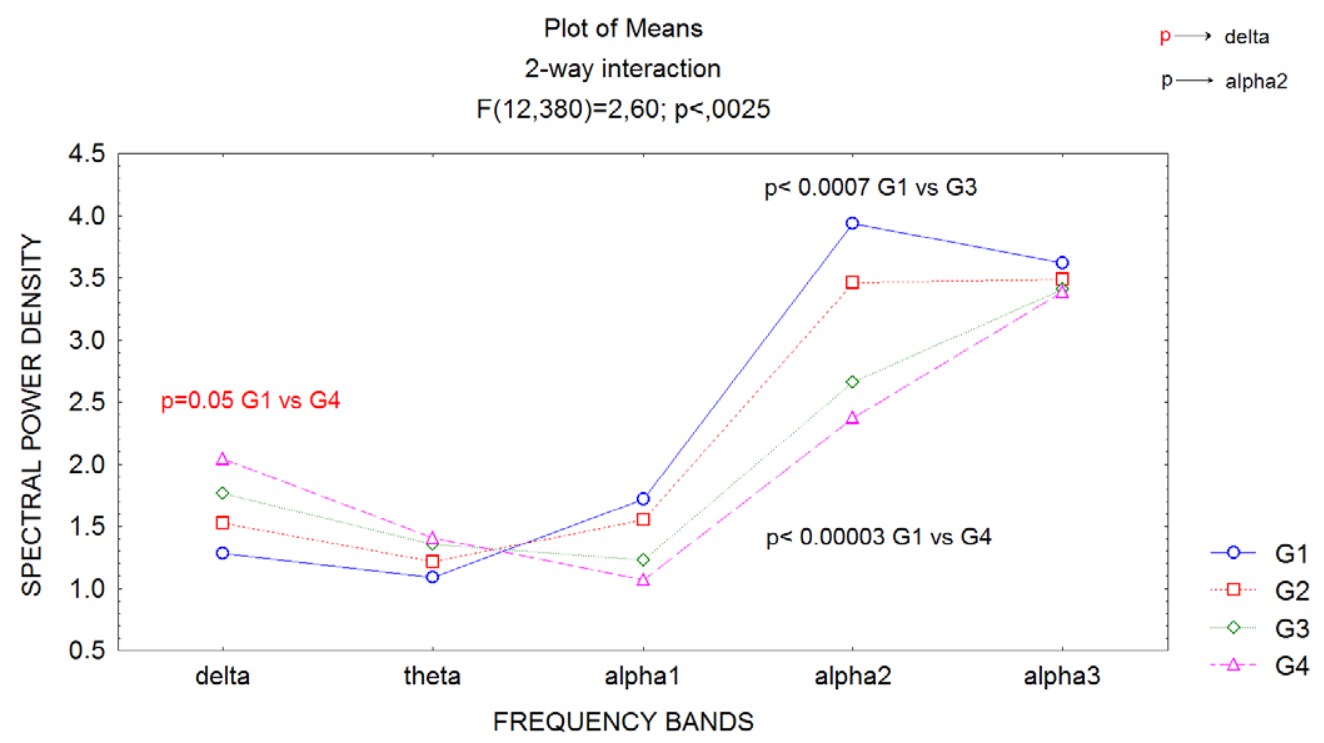

Fig. 2. Statistical ANOVA interaction among groups, factor and relative band power (delta, theta, alpha1, alpha2, alpha3). In the diagram the difference in delta and alpha2 power among groups is also indicated, based on Duncan's post-hoc testing. (G1, group 1) no vascular damage; (G2, group 2) mild vascular damage; (G3, group 3) moderate vascular damage; (G4, group 4) severe vascular damage.

results obtained in the principal analysis performed with five power bands. Finally, the post-hoc analysis did not show any significant difference in the $\delta$ power.

\section{Discussion}

\subsection{Methodological remarks}

Our results show a high correlation between the presence and amount of subcortical vascular damage and the examined EEG markers in both frequency and spectral power density domains. This particular aspect was suggested by a previous study (Moretti et al., 2004). Anyway, in that study the main patient selection criterion was the clinical diagnosis. In fact, the study compared normal elderly subjects with patients affected by Alzheimer's disease and vascular dementia. As a consequence, the observation of the $\mathrm{CV}$ damage impact on individual EEG markers was impaired, and the interpretation was speculative. We decided to verify the results in a better setting, by applying the $\mathrm{CV}$ damage as a principal selection criterion and thereby obtaining different groups within the study population.

Table 3

Mean values \pm standard error of $\theta / \alpha 1, \alpha 1 / \alpha 2, \alpha 2 / \alpha 3$ ratios in the MCI subgroups

\begin{tabular}{lllll}
\hline & Group 1 & Group 2 & Group 3 & Group 4 \\
\hline$\theta / \alpha 1$ & $0.7( \pm 0.05)$ & $0.77( \pm 0.05)$ & $1.17( \pm 0.05)$ & $1.39( \pm 0.14)$ \\
$\alpha 1 / \alpha 2$ & $0.46( \pm 0.03)$ & $0.5( \pm 0.03)$ & $0.53( \pm 0.05)$ & $0.47( \pm 0.04)$ \\
$\alpha 2 / \alpha 3$ & $1.27( \pm 0.12)$ & $1.16( \pm 0.1)$ & $0.85( \pm 0.05)$ & $0.79( \pm 0.07)$ \\
\hline
\end{tabular}

Group 1, no vascular damage; group 2, mild vascular damage; group 3, moderate vascular damage; group 4 , severe vascular damage.
We chose a cohort of MCI subjects as study population mainly for two reasons. The first reason addressed methodological issues. In fact, in a cohort of MCI patients, a greater variety of samples is available with respect to the $\mathrm{CV}$ damage. Naturally, it should be possible to enroll healthy elderly people. Such choice would be advantageous, given the homogeneity of the sample, but very probably it would reduce the opportunity to further subdivide the cohort based on the $\mathrm{CV}$ damage load. The choice of the MCI population allowed both these possibilities to coexist. Moreover, such choice is homogeneous with respect to the global cognitive state, and ensures the presence of larger samples in the sub-groups. As a consequence, these larger samples increase the reliability and sensitivity of results.

The second reason addressed an issue of clinical importance. We attempted to find EEG neurophysiological markers for CV damage. We would point out that the present study considers very global measures, such as averaged $\alpha$ frequency markers and spectral density power related to the total score on an ARWMC scale. Therefore, these results have to be interpreted as general parameters of EEG changes based on the CV damage. Once these markers have been confirmed in future studies, it will be possible to use them together with the existing tools based on neuroimaging or neuropsychological evaluations and, hence, to contribute to the differential diagnosis at an early stage of cognitive decline. So, we considered very global measures in order to obtain a tool easy to use in clinical practice.

\subsection{EEG frequency indices of $\alpha$ rhythms and $C V$ damage}

Our results show that the CV damage is strongly associated with TF modifications, possibly with a progressive 


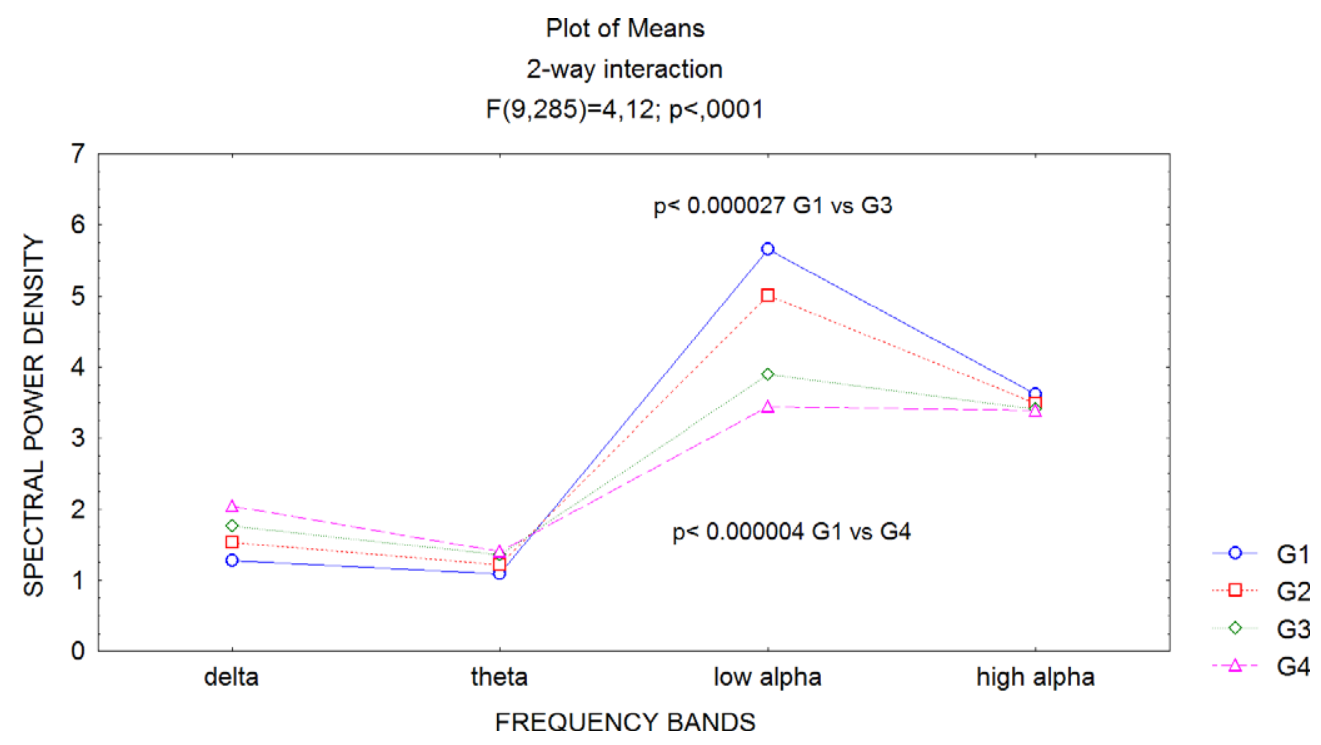

Fig. 3. Statistical ANOVA interaction among groups, factors (G1, G2, G3, G4), and relative band powers $(\delta, \theta$, low $\alpha$, high $\alpha$ ). In the diagram the difference in $\delta$ and $\alpha 2$ powers among groups is also indicated, based on Duncan's post-hoc testing. (G1, group 1) No vascular damage; (G2, group 2) mild vascular damage; (G3, group 3) moderate vascular damage; (G4, group 4) severe vascular damage.

frequency reduction that correlates with the $\mathrm{CV}$ damage in the four sub-groups of MCI patients. Although we did not find a main group effect when the TF was analyzed as the only dependent variable, the significant interaction between the Group factor and both IAF and TF was driven by the difference in the TF among groups. TF and IAF are undoubtedly different measures for the EEG analysis. It is to be noted that they both are expression of the dominant rhythm, and that it is of scientific interest to consider them together in order to better characterize the $\alpha$ frequency. The TF decrease was statistically significant across the group without $\mathrm{CV}$ damage, as compared to all the other groups, as well as between the groups with mild and severe CV damage. In fact, we observed a slowing of the $\alpha$ frequency in the two groups with greater CV damage, as compared to the groups with lesser $\mathrm{CV}$ damage. This is in line with a previous study (Moretti et al., 2004) showing that the major effect of the $\mathrm{CV}$ damage, in patients with vascular dementia (VaD) vs normal elderly and Alzheimer's patients, correlates with TF reduction. In addition, the significant negative correlation between $\mathrm{CV}$ damage score on ARWMC and TF confirmed this relation. A reasonable (although speculative) explanation of the present results is that the CV damage-induced slowing of the $\alpha$ frequency start point could be mainly attributed to the lowering of the conduction time of synaptic action potentials throughout cortico-subcortical fibers, such as cortico-basal or cortico-thalamic pathways (Tomasch, 1954; Steriade and Llinas, 1988). In fact, experimental models have previously shown that the EEG frequency is due to axonal delay and synaptic time of cortico-subcortical interactions (Lopes da Silva et al., 1976; Nunez et al., 2001; Doiron et al., 2003). Most interestingly, other studies have demonstrated that fiber myelination affects the speed propagation along cortical fibers, and that this parameter is strictly correlated to the frequency range recorded on the scalp. In fact, a theoretical model considering a mean speed propagation in white matter fibers of $7.5 \mathrm{~m} / \mathrm{s}$ (together with other parameters) is associated with a fundamental mode frequency of $9 \mathrm{~Hz}$ (Nunez, 1995; Nunez and Srinivasan, 2006), that is, the typical mode of scalp-recorded EEG. It is to be noted that a correlation between white matter damage and widespread slowing of EEG rhythmicity was found in other studies, following the presence of cognitive decline (d'Onofrio et al., 1996; Szelies et al., 1992, 1999), multiple sclerosis (Leocani et al., 2000), or cerebral tumors (Gloor et al., 1977; Goldensohn, 1979).

In the present study, we found neither impact of vascular damage on the IAF, nor significant correlation between $\mathrm{CV}$ damage score and IAF. On the contrary, in our previous study (Moretti et al., 2004), we found a slowing of the peak $\alpha$ frequency in patients with $\mathrm{VaD}$. This difference could be due to the disease stage. In fact, $\mathrm{VaD}$ patients are generally at a later stage of cognitive decline than MCI patients, and it is widely accepted that the subcortical vascular disease has a progressive course in the ultrastructural nature of lesions, as well as in the global load of CV damage (Frisoni et al., 2002). So, the IAF slowing could represent a subsequent change, due to a greater involvement of white matter pathways, during the course of disease. In this view, the TF could be a very sensitive marker for $\mathrm{CV}$ damage at an early stage of the disease. An alternative explanation has to consider the $\mathrm{CV}$ damage localization, that could be essential for the changes in EEG rhythmicity. CV lesions in the thalamocortical pathway could affect preferentially the oscillatory activity of the $\alpha$ frequency peak (Steriade and Llinas, 1988; Llinas et al., 1999; Jellinger, 2002, 2005), whereas lesions in cortico-basal 
or cortico-septal pathways could affect preferentially the oscillatory activity of the transition frequency between $\alpha$ and $\theta$ frequency bands (Colom, 2006). Obviously, given the correlative nature of this study, these conclusions have to be considered with caution. Further studies need to specify the causal nature of EEG changes in subjects with CV damage.

\subsection{Relative power spectral density and $C V$ damage}

As regards the spectral power, our results showed that the $\mathrm{CV}$ damage affected both $\delta$ and low $\alpha$ band power $(\alpha 1$ and $\alpha 2$ ). According to the existing literature (Nuwer, 1988; Leuchter et al., 1993), we analyzed the relative power band values for two reasons: (1) these were not affected by the influences of the head volume conductor; and (2) presented a lower group variability than the absolute power band values.

In the $\delta$ band we observed a power increase proportional to the $\mathrm{CV}$ damage, with a significant increase in the group with severe CV damage, as compared to the no-CV-damage group. The impact of the CV damage on the $\delta$ power was confirmed by the significant positive correlation between $\mathrm{CV}$ damage score and $\delta$ power itself. The increase in the $\delta$ band power could be explained as a progressive cortical disconnection due to the slowing of the conduction along cortico-subcortical connecting pathways. This result confirmed the increase in the $\delta$ band power we had observed in $\mathrm{VaD}$ patients, as compared to normal elderly subjects (Moretti et al., 2004). It is to be noted that the increase in the $\delta$ band power reflects a global state of cortical deafferentation, due to various anatomofunctional substrates, such as stages of sleep, metabolic encephalopathy or cortico-thalamocortical dysrhythmia (Llinas et al., 1999).

As regards the $\theta$ band power, we observed neither significant differences between groups, nor a significant correlation with the CV damage score. This is very surprising, given that the previous results (Moretti et al., 2004) showed an increase in the $\theta$ band power in $\mathrm{VaD}$ patients, but not in AD patients, as compared to normal elderly patients. The difference is probably due to a minor axonal damage in MCI patients who are in an early stage of disease. There is body of evidence that, initially, the CV damage involves astroglial cells and induces perivascular vacuolization (Jellinger, 2005). Only later an aggressive loss of the myelination process in the axonal structure becomes evident. Therefore, a $\theta$ band power increase significantly based on the CV damage could occur when the axonal destructuration reaches a well-defined stage during the course of the disease. Alternatively, a pathological increase of the $\theta$ band power could be due to a specific vascular impairment of the anatomical network ascending from the brainstem towards the medial septum-hippocampal formation involved in the generation of the $\theta$ rhythm (Colom, 2006).

With respect to the $\alpha$ band power, we would make a preliminary consideration. We found that the low $\alpha$ bandwidth differed in the $\alpha 1$ and $\alpha 2$ frequency bands among the groups. In the group without $\mathrm{CV}$ damage, as well as in the group with severe $\mathrm{CV}$ damage, the low $\alpha$ bandwidth was slightly narrower than in mild and moderate $\mathrm{CV}$ damage groups. This result deserves to be discussed, since previous studies (Jonkmann et al., 1992; Salansky et al., 1998) have demonstrated that small differences $(0.3 \mathrm{~Hz})$ in the EEG bandwidth could affect the estimation of the band power magnitude. It should be noted first that the statistical analysis did not show a significant difference among bandwidths. Moreover, in the present study the low $\alpha$ band was divided into two sub-bands ( $\alpha 1$ and $\alpha 2)$, so that the mean difference between the $\alpha$ sub-bandwidths was practically negligible. Furthermore, the band power was computed as the mean, but not the sum, of the power contained in each of the frequency bins within that band. Therefore, the bandwidth per se could not affect the estimation of the band power.

In the low $\alpha$ band power, we observed a significant decrease in the $\alpha 2$ band power for the groups with moderate and severe $\mathrm{CV}$ damage, as compared to the no-CV-damage group. In the $\alpha 1$ frequency band, there was a similar decrease although it did not reach statistically significant values. These results were confirmed by a correlation analysis which showed a significant negative correlation between $\mathrm{CV}$ damage score and $\alpha 1$ and $\alpha 2$ band powers. Moreover, the statistical analysis performed by pooling $\alpha 1$ and $\alpha 2$ frequency bands into a low $\alpha$ frequency band confirmed the globally homogeneous outcome profile for the low $\alpha$ power. The decrease in the $\alpha$ band power has been previously related to cholinergic deafferentation, both in animal (Holschneider et al., 1998) and human model studies (Moretti et al., 2004; Babiloni et al., 2006a,b). A cholinergic deficit is typical of AD patients, in whom it has been related to degenerative neuronal loss in basal forebrain (Sarter and Bruno, 1997, 1999, 2000). However, a cholinergic deficit could also occur in patient with subcortical CV damage, due to the damage of the cholinergic corticopetal pathways that run in the white matter (Erkinjuntti, 2002; Black et al., 2003). Recent studies have specifically addressed these issues (Pawlak and Krejza, 2005; Bocti et al., 2005) and shown that strategically located white matter hyperintensities could be related to the anatomical tracks of the cholinergic pathways. Thus, our results of a decrease - proportional to the vascular damage - in the low $\alpha$ band power could be related to the involvement of this pathway prone to vascular accidents targeting the subcortical white matter (Selden et al., 1998; Tomimoto et al., 2005).

In our results, the $\mathrm{CV}$ damage did not show any impact on the $\alpha 3$ (or high $\alpha$ ) power. This is a confirmation of what we found in the previous study, where no differences between $\mathrm{VaD}$ patients and normal elderly (but not in AD vs normal elderly) subjects were detected in the $\alpha 3$ power. Together, these results could suggest different generators for low $\alpha$ and high $\alpha$ frequency bands. In particular, the low $\alpha$ band power could affect cortico-subcortical 
mechanisms, such as cortico-thalamic, cortico-striatal and cortico-basal ones. This could explain the sensitivity of the low $\alpha$ frequency band to subcortical vascular damage. On the contrary, the $\alpha 3$ band power could affect to a greater extent those cortico-cortical interactions based on synaptic efficiency prone to degenerative rather than $\mathrm{CV}$ damages (Klimesch, 1999; Klimesch et al., 2007).

\subsection{EEG relative power ratios and $C V$ damage}

In order to find reliable indices of CV damage, we checked the $\theta / \alpha 1$ band power ratio. Previous studies have shown the reliability of this kind of approach in quantitative EEG in demented patients (Jelic et al., 1997). The importance of this ratio lies in the presence of such frequency bands on the opposite side of the TF, that is, the EEG frequency index most significantly affected by the $\mathrm{CV}$ damage. So, the $\theta / \alpha 1$ band power ratio could represent the most sensitive EEG marker of $\mathrm{CV}$ damage. The results showed a significant increase of the $\theta / \alpha 1$ band power ratio in moderate and severe $\mathrm{CV}$ damage groups, as compared to mild and no-CV-damage groups. This ratio increase establishes a proportional increase of the $\theta$ band power relative to the $\alpha 1$ band power with respect to the CV damage, even though a significant increase in the $\theta$ band power per se (or a decrease in the $\alpha 1$ band power per se) is not present. This could suggest a reliable specificity for the $\theta / \alpha 1$ band power ratio in focusing the presence of a subcortical $\mathrm{CV}$ damage. Noteworthily, future studies will address the reliability of this index not only with respect to groups, but also at individual level and for individual cortical regions. The $\alpha / \theta$ band power ratio has been evaluated in a recent study (Jelic et al., 1997). In this study, the $\alpha / \theta$ band power ratio in a sample of patients with Alzheimer's disease was significantly reduced, as compared with healthy controls. It is not possible to make direct comparisons with this study, in that fixed frequency bands $(\theta$ and $\alpha$ ) were considered. Moreover, the focus of the cited work was not the CV damage. Nevertheless, given the presence of vascular involvement also in $\mathrm{AD}$ patients, it could be possible that the decrease in the $\alpha / \theta$ band power ratio is - at least, partly attributable to a subcortical vascular component.

Our results show that a different relation exists between $\mathrm{CV}$ damage and $\alpha 2$ and $\alpha 3$ band power. In order to better detect such difference, we tested the $\alpha 2 / \alpha 3$ band power ratio and, as a control, the $\alpha 1 / \alpha 2$ band power ratio. The rationale was that, if there emerged a real difference, the first ratio, describing a different behaviour between low and high $\alpha$ frequency bands, would show a significant difference between groups, while no difference would arise in the latter ratio, representing only the low $\alpha$ band power. The results confirmed this hypothesis. The $\alpha 2 / \alpha 3$ band power ratio showed a significant main group effect, with a decrease in no-CV-damage vs moderate and severe $\mathrm{CV}$ damage groups, and in mild CV damage $v s$ severe CV damage groups. This is due to the stability of the $\alpha 3$ band power even in the groups with greater amount of $\mathrm{CV}$ damage, probably due to the relative sparing of cortical neuronal reserve. Moreover, the ratio relevant to the low $\alpha$ frequency did not reveal any significant difference. This confirms a different behaviour with respect to the $\mathrm{CV}$ damage and a different level of generation of different $\alpha$ frequency bands.

\section{Conclusions}

The EEG analysis confirmed a good reliability in detecting changes that correlated with subcortical vascular damage. Some indices $(\theta / \alpha 1$ and $\alpha 2 / \alpha 3$ band power ratio) appear to be particularly reliable in order to discriminate between degenerative and subcortical vascular forms of cognitive decline, even in the early stage of the disease. Further studies have to show such reliability in single cases. In particular, much effort is necessary to enucleate the specific EEG rhythmicity changes due to vascular or degenerative impairment. Above all, a detailed regional analysis of the EEG source generators could highlight closer relations among cerebral oscillatory activity, CV damage and localization of $\mathrm{CV}$ lesions.

\section{References}

Albert M, Smith LA, Scherr PA, Taylor JO, Evans DA, Funkenstein HH. Use of brief cognitive tests to identify individuals in the community with clinically diagnosed Alzheimer's disease. Int J Neurosci 1991;57(3-4):167-78.

Amodio P, Wenin H, Del Piccolo F, Mapelli D, Montagnese S, Pellegrini A, et al. Variability of trail making test, symbol digit test and line trait test in normal people. A normative study taking into account agedependent decline and sociobiological variables. Aging Clin Exp Res 2002;14:117-31.

Babiloni C, Binetti G, Cassetta E, Dal Forno G, Del Percio C, Ferreri F, et al. Sources of cortical rhythms change as a function of cognitive impairment in pathological aging: a multi-centric study. Clin Neurophysiol 2006a;117(2):252-68.

Babiloni C, Frisoni G, Steriade M, Bresciani L, Binetti G, Del Percio C, et al. Frontal white matter volume and $\delta$ EEG sources negatively correlate in awake subjects with mild cognitive impairment and Alzheimer's disease. Clin Neurophysiol 2006b;117:1113-29.

Basso A, Capitani E, Laiacona M. Raven's coloured progressive matrices: normative values on 305 adult normal controls. Funct Neurol 1987;2:189-94.

Bennys K, Rondouin G, Vergnes C, Touchon J. Diagnostic value of quantitative EEG in Alzheimer's disease. Neurophysiol Clin 2001;31:153-60.

Black S, Roman GC, Geldmacher DS, Salloway S, Hecker J, Burns A, et alDonepezil 307 Vascular Dementia Study Group. Efficacy and tolerability of donepezil in vascular dementia: positive results of a 24week, multicenter, international, randomized, placebo-controlled clinical trial. Stroke 2003;34(10):2323-30.

Bocti C, Swartz RH, Gao FQ, Sahlas DJ, Behl P, Black Sandra E. A New Visual Rating Scale to assess strategic white matter hyperintensities within cholinergic pathways in dementia. Stroke 2005;36:2126-131.

Burton E, Ballard C, Stephens S, Kenny RA, Kalaria R, Barber R, et al. Hyperintensities and fronto-subcortical atrophy on MRI are substrates of mild cognitive deficits after stroke. Dement Geriatr Cogn Disord 2003;16:113-8.

Caffarra P, Vezzadini G, Dieci F, Zonato F, Venneri A. Rey-Osterrieth complex figure: normative values in an Italian population sample. Neurol Sci 2002;22:443-7.

Carlesimo GA, Caltagirone C, Gainotti G. The mental Deterioration Battery: normative data, diagnostic reliability and qualitative analyses 
of cognitive impairment. The Group for the Standardization of the Mental Deterioration Battery. Eur Neurol 1996;36:378-84.

Colom LV. Septal networks: relevance to $\theta$ rhythm, epilepsy and Alzheimer's disease. J Neurochem 2006;96:609-23.

Destexhe A, Sejnowski TJ. Synchronized oscillations in thalamic networks: insight from modeling studies. In: Steriade M, Jones EG, McCormick DA, editors. Thalamus. Amsterdam: Elsevier; 1996.

Devanand DP, Folz M, Gorlyn M, Moeller JR, Stern J. Questionable dementia: clinical course and predictors of outcome. J Am Geriatr Soc 1997;45:321-8.

Doiron B, Chacron MJ, Maler L, Longtin L, Bastian J. Inhibitory feedback required for network oscillatory responses to communication but not prey stimuli. Nature 2003;421:538-43.

d'Onofrio S, Salvia S, Petretta V, Bonavita V, Rodriguez G, Tedeschi G. Quantified-EEG in normal aging and dementias. Acta Neurol Scand 1996;93(5):336-45.

Elul R. The genesis of EEG. Int Rev Neurobiol 1972;15:227-72.

Erkinjuntti T. Cognitive decline and treatment options for patients with vascular dementia. Acta Neurol Scand Suppl 2002;178:15-8.

Erkinjuntti T, Kurz A, Gauthier S, Bullock R, Lilienfeld S, Damaraju CV. Efficacy of galantamine in probable vascular dementia and Alzheimer's disease combined with CV disease: a randomised trial. Lancet 2000;359:1283-90.

Fein G, Di Sclafani V, Tanabe J, Cardenas V, Weiner MW, Jagust WJ, et al. Hippocampal and cortical atrophy predict dementia in subcortical ischemic vascular disease. Neurology 2000;55:1626-35.

Flicker CS, Ferris H, Reisberg B. Mild cognitive impairment in the elderly: predictors of dementia. Neurology 1991;41:1006-9.

Folstein MF, Folstein SE, McHugh PR. 'Mini mental state': a practical method for grading the cognitive state of patients for clinician. J Psychiatr Res 1975;12:189-98.

Frisoni GB, Galluzzi S, Bresciani L, Zanetti O, Geroldi C. Mild cognitive impairment with subcortical vascular features: Clinical characteristics and outcome. J Neurol 2002;249:1423-32.

Galluzzi S, Sheu CF, Zanetti O, Frisoni GB. Distinctive clinical features of mild cognitive impairment with subcortical CV disease. Dement Geriatr Cogn Disord 2005;19:196-203.

Geroldi C, Rossi R, Calvagna C, Testa C, Bresciani L, Binetti G, et al. Medial temporal atrophy but not memory deficit predicts progression to dementia in patients with mild cognitive impairment. J Neurol Neurosurg Psychiatry 2006;77:1219-22.

Gloor P, Ball G, Schaul N. Brain lesions that produce $\delta$ waves in the EEG. Neurology 1977;27(4):326-33.

Goldensohn ES. Use of EEG for evaluation of focal intracranial lesions. In: Klass DW, Daly DD, editors. Current practice of clinical electroencephalography. New York: Raven; 1979. p. 307-41.

Gorelick PB. Prevention. In: Bowler JV, Hachinski V, editors. Vascular cognitive impairment. Preventable dementia. Oxford: Oxford University Press; 2003. p. 308-20.

Gueguen B, Derouesne C, Bourdel MC, Guillou S, Landre E, Gaches J, et al. Apport de $1^{\prime} E E G$ quantifiee au diagnostic de demence de type Alzheimer. Neurophysiol Clin 1991;21:357-71.

Hentschel F, Kreis M, Damian M, Krumm B. Microangiopathic lesions of white matter. Quantitation of cerebral MRI findings and correlation with psychological tests. Nervenarzt 2003;74:355-61.

Holschneider DP, Leuchter AF, Scremin OU, Treiman DM, Walton NW. Effects of cholinergic deafferentation and NGF on brain electrical coherence. Brain Res Bull 1998;45:531-41.

Huang C, Wahlund LO, Dierks T, Julin P, Winblad B, Jelic V. Discrimination of Alzheimer's disease and mild cognitive impairment by equivalent EEG sources: a cross-sectional and longitudinal study. Clin Neurophysiol 2000;11:1961-7.

Hughes CP, Berg L, Danziger WL, Cohen LA, Martin RL. A new clinical rating scale for the staging of dementia. $\mathrm{Br} \mathrm{J}$ Psychiatry 1982;140:1225-30.

Jelic V, Shigeta M, Julin P. Quantitative electroencephalography power and coherence in Alzheimer's disease and mild cognitive impairment. Dementia 1996;7:314-23.
Jelic V, Julin P, Shigeta M, Nordberg A, Lannfelt L, Winblad B, et al. Apolipoprotein E $\varepsilon 4$ allele decreases functional connectivity in Alzheimer's disease as measured by EEG coherence. J Neurol Neurosurg Psychiatry 1997;63(1):59-65.

Jelic V, Johansson SE, Almkvist O, Shigeta M, Julin P, Nordberg A, et al. Quantitative electroencephalography in mild cognitive impairment: longitudinal changes and possible prediction of Alzheimer's disease. Neurobiol Aging 2000;21(4):533-40.

Jellinger KA. The pathology of ischemic-vascular dementia: an update. $\mathbf{J}$ Neurol Sci 2002;203-204:153-7.

Jellinger KA. The neuropathological substrate of vascular-ischemic dementia. In: Paul RH, Cohen R, Ott BR, Salloway S, editors. Vascular dementia - CV mechanisms and clinical management. Totowa: NJ7 Humana Press; 2005. p. 23-56.

Jonkmann E, De Weend A, Poortvliet DCJ. EEG studies in workers exposed to solvents or pesticides. Electroenceph clin Neurophysiol 1992;82:438-44.

Kimura S, Saito H, Minami M, Togashi H, Nakamura N, Nemoto M, et al. Pathogenesis of vascular dementia in stroke-prone spontaneously hypertensive rats. Toxicology 2000;153:167-78.

Klimesch W. EEG- $\alpha$ rhythms and memory processes. Int J Psychophysiol 1997;26:319-40.

Klimesch W. EEG alpha and theta oscillations reflect cognitive and memory performance: a review and analysis. Brain Res Rev 1999;29:169-95.

Klimesch W, Sauseng P, Hanslmayr S. EEG alpha oscillations: The inhibition timing hypothesis. Brain Res Rev 2007;53:63-88.

Koenig T, Prichep L, Dierks T, Hubl D, Wahlund LO, John ER, et al. Decreased EEG synchronization in Alzheimer's disease and mild cognitive impairment. Neurobiol Aging 2005;26(2):165-71.

Kopruner V, Pfurtscheller G, Auer LM. Quantitative EEG in normals and in patients with cerebral ischemia. In: Pfurtscheller G, Jonkman EJ, Lopes da Silva FH, editors. Brain ischemia: quantitative EEG and imaging techniques. Progress in brain research 1984; vol. 62. Amsterdam: Elsevier; 1984. p. 29-50.

Kramer JH, Reed BR, Mungas D, Weiner MW, Chui HC. Executive dysfunction in subcortical ischaemic vascular disease. J Neurol Neurosurg Psychiatry 2002;72:217-20.

Lawton MP, Brodie EM. Assessment of older people: self maintaining and instrumental activity of daily living. J Gerontol 1969;9: 179-86.

Leocani L, Locatelli T, Martinelli V, Rovaris M, Falautano M, Filippi M, et al. Electroencephalographic coherence analysis in multiple sclerosis: correlation with clinical, neuropsychological, and MRI findings. J Neurol Neurosurg Psychiatry 2000;69:192-8.

Leuchter AF, Cook IA, Newton TF, Dunkin J, Walter DO, RosenbergThompson S, et al. Regional differences in brain electrical activity in dementia: use of spectral power and spectral ratio measures. Electroenceph clin Neurophysiol 1993;87:385-93.

Llinas RR, Ribary U, Jeanmonod D, Kronberg E, Mitra PP. Thalamocortical dysrhythmia: a neurological and neuropsychiatric syndrome characterized by magnetoencephalography. Proc Natl Acad Sci USA 1999;96(26):15222-7.

Lopes da Silva FH, van Rotterdam A, Barts P, van Heusden E, Burr W. Models of neuronal populations: the basic mechanism of rhythmicity. In: Corner MA, Swaab DF, editors. Perspectives of brain research. Progress in brain research 1976; vol. 45. Amsterdam: Elsevier; 1976. p. 281-308.

Lopes da Silva FH, Mooibroek J, van Rotterdam A. Relative contribution of intracortical and thalamo-cortical process in the generation of alpha rhythms revealed by partial coherence analysis. Electroenceph clin Neurophysiol 1980;50:449-56.

Maurer K, Dierks T. Functional imaging procedures in dementias: mapping of EEG and evoked potentials. Acta Neurol Scand 1992;139:40-6.

Moretti DV, Babiloni C, Binetti G, Cassetta E, Dal Forno G, Ferreri F, et al. Individual analysis of EEG frequency and band power in mild Alzheimer's disease. Clin Neurophysiol 2004;115:299-308. 
Niedermayer E. The normal EEG in waking adult. In: Niedermayer E, Lopes da Silva FH, editors. Electroencefalography: basic principles, clinical applications and related fields. Baltimore, MD: Williams and Wilkins; 1993. p. 131-52.

Novelli G, Papagno C, Capitani E, Laiacona M, Vallar G, Cappa SF. Tre test clinici di ricerca e produzione lessicale. Taratura su soggetti normali. Arch Psicolog Neuro Psichiatria 1986;4:477-505.

Nunez PL. Neocortical dynamics and human EEG rhythms. New York: Oxford University Press; 1995.

Nunez PL, Srinivasan R. A theoretical basis for standing and traveling brain waves. Clin Neurophysiol 2006;117(11):2425-35.

Nunez PL, Wingeier BM, Silberstein RB. Spatial-temporal structures of human alpha rhythms: theory, microcurrent sources, multiscale measurements, and global binding of local networks. Hum Brain Mapp 2001;13:125-64.

Nuwer MR. Quantitative EEG I: technique and problems of frequency analysis and topographic mapping. J Clin Neurophysiol 1988;5:1-43.

Pawlak M, Krejza J. A new visual scale to assess white matter hyperintensities within cholinergic pathways. Stroke 2005;36:2064-5.

Petersen RC, Smith GE, Ivnik RJ, Tangalos EG, Schaid SN, Thibodeau $\mathrm{SN}$, et al. Apolipoprotein E status as a predictor of the development of Alzheimer's disease in memoryimpaired individuals. J Am Med Assoc 1995;273:1274-8.

Petersen RC, Smith GE, Waring SC, Ivnik RJ, Kokmen E, Tangelos EG. Aging, memory, and mild cognitive impairment. Int Psychogeriatr 1997;9(Suppl. 1):65-9.

Petersen RC, Doody R, Kurz A, Mohs RC, Morris JC, Rabins PV, et al. Current concepts in mild cognitive impairment. Arch Neurol 2001;58(12):1985-92.

Pfurtscheller G, Lopes da Silva F. Event-related EEG/MEG synchronization and desynchronization: basic principles. Clin Neurophysiol 1999;110:1842-57.

Portet F, Ousset PJ, Visser PJ, Frisoni GB, Nobili F, Scheltens Ph, et al. The MCI Working Group of the European Consortium on Alzheimer's disease (EADC). Mild cognitive impairment (MCI) in medical practice: a critical review of the concept and new diagnostic procedure. Report of the MCI Working Group of the European Consortium on Alzheimer's disease. J Neurol Neurosurg Psychiatry 2006;77:714-18.

Prichep LS, John ER, Ferris SH, Rausch L, Fang Z, Cancro R, et al. Prediction of longitudinal cognitive decline in normal elderly with subjective complaints using electrophysiological imaging. Neurobiol. Aging 2006;27:471-81.

Radloff LS. The CES-D scale: a self-report depression scale for research in the general population. Appl Psycholog Measure 1977;1:385-401.

Rosen WG, Terry RD, Fuld PA, Katzman R, Peck A. Pathological verification of ischemic score in differentiation of dementias. Ann Neurol 1980;7(5):486-8.

Rossini PM, Del Percio C, Pasqualetti P, Cassetta E, Binetti G, Dal Forno $\mathrm{G}$, et al. Conversion from mild cognitive impairment to Alzheimer's disease is predicted by sources and coherence of brain electroencephalography rhythms. Neuroscience 2006;143:793-803.

Salansky N, Fedotchev A, Bondar A. Responses of the nervous system to low frequency stimulation and EEG rhythms: clinical implications. Neurosci Biobehav Rev 1998;22:395-409.
Sarter M, Bruno JP. Cognitive functions of cortical cholinergic: toward a unifying hypothesis. Brain Res Rev 1997;23:329-43.

Sarter M, Bruno JP. Abnormal regulation of corticopetal cholinergic neurons and impaired information processing in neuropsychiatric disorders. Trends Neurosci 1999;22:67-74.

Sarter M, Bruno JP. Cortical cholinergic inputs mediating arousal, attentional processing and dreaming: differential afferent regulation of the basal forebrain by telencephalic and brainstem afferents. Neuroscience 2000;95:933-52.

Schreiter-Gasser U, Gasser Th, Ziegler P. Quantitative EEG-analysis in early onset Alzheimer's disease: a controlled study. Electroenceph clin Neurophysiol 1993;86:15-22.

Selden NR, Gitelman DR, Salamon-Nurayama N, Parrish TB, Mesulam MM. Trajectories of cholinergic pathways within the cerebral hemispheres of human brain. Brain 1998;121:2248-57.

Shulman KI. Clock-drawing: is it the ideal cognitive screening test? Int J Geriatr Psychiatry 2000;15:548-61.

Singer W. Synchronization of cortical activity and its putative role in information processing and learning. Annu Rev Physiol 1993;55:349-74.

Spinnler H, Tognoni G. Standardizzazione e taratura italiana di test neuropsicologici. Ital J Neurol Sci 1987;6(Suppl. 8):1-120.

Steriade M, Llinas RR. The functional states of the thalamus and the associated neuronal interplay. Physiol Rev 1988;68:649-742.

Suffczynski P, Stilian K, Pfurtscheller G, Lopes da Silva FH. Computational model of thalamo-cortical networks: dynamical control of alpha rhythms in relation to focal attention. Int $\mathbf{J}$ Psychophysiol 2001;43:25-40.

Swartz RH, Black SE. How common is vascular compromise of cholinergic white matter pathways in a memory clinic sample? J Neurol Sci 2002;203-204:281.

Szelies B, Grond M, Herholz K, Kessler J, Wullen T, Heiss WD Quantitative EEG mapping and PET in Alzheimer's disease. J Neurol Sci 1992;110:46-56.

Szelies B, Mielke R, Kessler J, Heiss WD. EEG power changes are related with regional cerebral glucose metbolism in vascular dementia. Clin Neurophysiol 1999;110:615-20.

Tomasch J. Size, distribution and number of fibers in the human corpus callosum. Anat Rec 1954;119:119-35.

Tomimoto H, Ohtani R, Shibata M, Nakamura N, Ihara M. Loss of cholinergic pathways in vascular dementia of the Binswanger type. Dement Geriatr Cogn Disord 2005;19:282-8.

Wahlund LO, Barkhof F, Fazekas F, Bronge L, Augustin M, Sjögren M, et al. A new rating scale for age-related white matter changes applicable to MRI and CT. Stroke 2001;32:1318-22.

Wentzel C, Rockwood K, MacKnight C, Hachinski V, Hogan DB, Feldman $\mathrm{H}$, et al. Progression of impairment in patients with vascular cognitive impairment without dementia. Neurology 2001;57: 714-6.

Zappoli R, Versari A, Paganini M, Arnetoli G, Muscas GC, Gangemi PF, et al. Brain electrical activity (quantitative EEG and bit-mapping neurocognitive CNV components), psychometrics and clinical findings in presenile subjects with initial mild cognitive decline or probable Alzheimer-type dementia. Ital J Neurol Sci 1995;16(6):341-76. 